\title{
The Second Painlevé Hierarchy and the Stationary KdV Hierarchy ${ }^{\dagger}$
}

\author{
By
}

Nalini JosHi*

\begin{abstract}
It is well known that soliton equations such as the Korteweg-de Vries equation are members of infinite sequences of PDEs known as hierarchies. Here we consider infinite sequences of ODEs associated with the Painlevé equations. We review methods of constructing such hierarchies, specifically the second Painlevé hierarchy, as reductions of PDE hierarchies. We also show that in the large-parameter limit, the solutions of the second Painlevé hierarchy are given by the periodic solutions of the stationary KdV hierarchy.
\end{abstract}

\section{$\S 1$. Introduction}

The six classical Painlevé equations are integrable non-linear second-order ordinary differential equations (ODEs) that are intimately related to soliton equations [1]. The Painlevé equations were first discovered by Painlevé and his school [21] when they classified ODEs according to the complex analytic properties of their solutions. In particular, Painlevé was interested in equations that defined new transcendental functions globally in the complex plane. As part of this search, he looked for differential equations that possess what is now called the Painlevé property, i.e., all movable singularities of all solutions are poles. The six Painlevé equations represent the only possible canonical classes

Communicated by T. Kawai. Received December 10, 2003. Revised February 20, 2004. 2000 Mathematics Subject Classification(s): Primary 33E17; Secondary 34E05.

Key words and phrases: Painlevé equations, Painlevé Hierarchies, Asymptotics.

Research supported by Australian Research Council Discovery Grant \#DP0345505.

${ }^{\dagger}$ This article is an invited contribution to a special issue of Publications of RIMS commemorating the fortieth anniversary of the founding of the Research Institute for Mathematical Sciences.

* School of Mathematics and Statistics F07, University of Sydney, NSW2006 Sydney,

Australia.

e-mail: nalini@maths.usyd.edu.au

(C) 2004 Research Institute for Mathematical Sciences, Kyoto University. All rights reserved. 
of equations in a class of second-order ODEs that not only possess the Painlevé property but that also define new trancendental functions.

Recent interest in the Painlevé equations arose because they are similarity reductions of soliton equations. For example, the Korteweg-de Vries (KdV) equation

$$
U_{t}+6 U U_{x}+U_{x x x}=0
$$

has a similarity reduction given by

$$
U(x, t)=\frac{u(z)}{(3 t)^{\frac{2}{3}}}, \quad z=\frac{x}{(3 t)^{\frac{1}{3}}} .
$$

The resulting third-order ODE for $u(z)$

$$
u^{\prime \prime \prime}(z)+6 u^{\prime}(z) u(z)-z u^{\prime}(z)-2 u(z)=0
$$

can be integrated by using the transformation

$$
u(z)=V_{z}-V^{2},
$$

where $V(z)$ solves the second Painlevé equation

$$
\mathrm{P}_{\mathrm{II}}: \quad V^{\prime \prime}=2 V^{3}+z V+\alpha, V=V(z), \alpha \text { const. }
$$

It is well known from soliton theory that equations such as the KdV can be solved (for large classes of initial data) through their associated linear problems (called Lax pairs) for which they act as compatibility conditions. By reduction, the Painlevé equations also possess associated linear problems. Their initial value problems have been shown to be solvable through the Riemann-Hilbert transform method applied to such linear problems [7].

For each soliton equation, such as the KdV equation, there exists an infinite sequence of PDEs, indexed by order, with that equation as the first member. Symmetry reductions of such PDE hierarchies lead to ODE hierarchies with the Painlevé equations as first members. The second Painlevé hierarchy is given recursively by

$$
\mathrm{P}_{\mathrm{II}}^{(n)}: \quad\left(\frac{d}{d z}+2 V\right) \mathcal{L}_{n}\left\{V_{z}-V^{2}\right\}=z V+\alpha_{n}, n \geq 1
$$

where $\alpha_{n}$ are constants and $\mathcal{L}_{n}$ is the operator defined by

$$
\partial_{z} \mathcal{L}_{n+1}\{U\}=\left(\partial_{z z z}+4 U \partial_{z}+2 U_{z}\right) \mathcal{L}_{n}\{U\}
$$




$$
\mathcal{L}_{1}\{U\}=U
$$

In Section 2, we review the construction of this hierarchy by a reduction of the $\mathrm{KdV}$ hierarchy. We also show how to derive the linear problem(s) associated with the second Painlevé hierarchy from that of the KdV hierarchy.

The KdV equation is an infinite-dimensional completely integrable Hamiltonian system. Its hierarchy also forms a completely integrable Hamiltonian system [19], with an infinite set of conserved, functionally independent Hamiltonians that are in involution. Since the equations of the KdV hierarchy all share these conserved quantities, the solutions of the KdV equation can be said to describe the solutions of the whole hierarchy.

However, this involutive structure breaks down for the Painlevé hierarchies. The members of the reduced hierarchy no longer share all their conserved quantities. Moreover, it is not clear whether the tools used for solving the second-order Painlevé equations work for the whole hierarchy. For example, the Riemann-Hilbert transform crucial for solving the Painlevé equations appears to have only been proved to be invertible for the second-order cases. These considerations make the development of any tools that describe solutions of the whole Painlevé hierarchy valuable.

Another open question is whether the higher order members of the hierarchy admit solutions that are more transcendental than the lower-order members. If the asymptotic behaviours of the solutions of the $n$-th equation are more transcendental than those of the solutions of lower equations in the hierarchy, then it follows that the corresponding solutions of the $n$-th equation must be more transcendental that those of the lower ones. To resolve this question, we considered limiting behaviours of solutions of the second Painlevé hierarchy $\mathrm{P}_{\mathrm{II}}^{(n)}$ as $\alpha_{n} \rightarrow \infty$. In Section 3 , we show that in this limit $\mathrm{P}_{\mathrm{II}}^{(n)}$ becomes a transformed version of the stationary KdV hierarchy.

It is well known that stationary periodic solutions of the $\mathrm{KdV}$ equation and its hierarchy may be constructed through its associated linear problem. We review this construction in Section 4. These results show that, in the large parameter limit, the solutions of the second Painlevé hierarchy are given by elliptic and hyperelliptic solutions of the stationary KdV hierarchy and that the genus of the hyperelliptic functions increases with $n$. Since hyperelliptic functions cannot be expressed as algebraic functions (or compositions) of elliptic functions, these results imply that the solutions of the higher order members of $\mathrm{P}_{\mathrm{II}}^{(n)}$ necessarily must be more transcendental that those of $\mathrm{P}_{\mathrm{II}}$. We end the paper with a summary in Section 5 . 


\section{§2. A Second Painlevé Hierarchy}

In this section, we review the method of deducing the Painlevé hierarchy $\mathrm{P}_{\mathrm{II}}^{(n)}$ as a reduction of the KdV hierarchy. Although this reduction is well known, the detailed calculations and the integration leading to the Painlevé hierarchy do not appear to be given explicitly in the literature. Similarly, the construction of the linear problem associated with each member of a Painlevé hierarchy is not completely explicit in the literature. We give the hierarchy of linear problems associated with the KdV hierarchy and show how its explicit reduction leads to the linear problems associated with the Painlevé hierarchy. The linear problems are important for the deduction of asymptotic behaviours.

\section{§2.1. KdV hierarchy}

The KdV hierarchy is given by

$$
U_{t_{2 n+1}}+\partial_{x} \mathcal{L}_{n+1}[U]=0, \quad n \geq 0
$$

where $\mathcal{L}_{n}$ satisfies the recursion relation (1.4a), with $z$ now replaced by $x$, and $\mathcal{L}_{0}=\frac{1}{2}$. The first few members of this sequence are

$$
\begin{aligned}
& n=0: \quad \partial_{x} \mathcal{L}_{1}=\left(\partial_{x}^{3}+4 U \partial_{x}+2 U_{x}\right)\left(\frac{1}{2}\right)=U_{x} \Rightarrow\left\{\begin{array}{l}
U_{t_{1}}+U_{x}=0 \\
\mathcal{L}_{1}=U
\end{array}\right. \\
& n=1: \quad \partial_{x} \mathcal{L}_{2}=\left(\partial_{x}^{3}+4 U \partial_{x}+2 U_{x}\right)(U) \\
&= U_{x x x}+4 U U_{x}+2 U_{x} U \Rightarrow\left\{\begin{array}{l}
U_{t_{3}}+6 U U_{x}+U_{x x x}=0 \\
\mathcal{L}_{2}=U_{x x}+3 U^{2}
\end{array}\right. \\
& n=2: \quad \partial_{x} \mathcal{L}_{3}=\left(\partial_{x}^{3}+4 U \partial_{x}+2 U_{x}\right)\left(U_{x x}+3 U^{2}\right) \\
&= U_{x x x x x}+\partial_{x x}\left(6 U U_{x}\right)+4 U U_{x x x}+4 U\left(6 U U_{x}\right) \\
&+2 U_{x} U_{x x}+6 U_{x} U^{2} \\
&= U_{5 x}+6 U_{x x} U_{x}+12 U_{x} U_{x x}+6 U U_{x x x} \\
& \quad+2 U_{x} U_{x x}+4 U U_{x x x}+30 U^{2} U_{x}
\end{aligned}
$$


The fact that at each step equation (1.4a) can be integrated to obtain the differential operator $\mathcal{L}_{n+1}$ was proved in [18].

\section{§2.2. MKdV hierarchy}

Under the Miura map $U=W_{x}-W^{2}$, the KdV equation becomes

$$
\begin{gathered}
\partial_{t}\left(W_{x}-W^{2}\right)+6\left(W_{x}-W^{2}\right)\left(W_{x x}-2 W W_{x}\right)+W_{x x x x} \\
-2 W_{x x} W_{x}-4 W_{x} W_{x x}-2 W W_{x x x}=0
\end{gathered}
$$

This implies

$$
\left(\partial_{x}-2 W\right) W_{t}-6\left(\partial_{x}-2 W\right)\left(W^{2} W_{x}\right)+\left(\partial_{x}-2 W\right)\left(W_{x x x}\right)=0,
$$

which in turn can be rewritten as

$$
\left(\partial_{x}-2 W\right)\left(W_{t}-6 W^{2} W_{x}+W_{x x x}\right)=0
$$

Therefore, if $W(x, t)$ satisfies the modified $\mathrm{KdV}(\mathrm{MKdV})$ equation

$$
W_{t}-6 W^{2} W_{x}+W_{x x x}=0
$$

then $U=W_{x}-W^{2}$ satisfies the $\mathrm{KdV}$ equation. From the $\mathrm{KdV}$ hierarchy we get

$$
\partial_{t_{2 n+1}}\left(W_{x}-W^{2}\right)+\partial_{x} \mathcal{L}_{n+1}\left[W_{x}-W^{2}\right]=0
$$

but

$$
\begin{aligned}
\partial_{x} \mathcal{L}_{n+1}= & \left(\partial_{x}^{3}+4\left(W_{x}-W^{2}\right) \partial_{x}+2 W_{x x}-4 W W_{x}\right) \mathcal{L}_{n} \\
= & \left(\partial_{x}-2 W\right)\left(\partial_{x x} \mathcal{L}_{n}\right)+2 W \partial_{x x} \mathcal{L}_{n} \\
& +4 W_{x} \partial_{x} \mathcal{L}_{n}-4 W^{2} \partial_{x} \mathcal{L}_{n}+\left(2 W_{x x}-4 W W_{x}\right) \mathcal{L}_{n} \\
= & \left(\partial_{x}-2 W\right)\left(\partial_{x x} \mathcal{L}_{n}+2 W \partial_{x} \mathcal{L}_{n}\right) \\
& +2 W_{x} \partial_{x} \mathcal{L}_{n}+2 W_{x x} \mathcal{L}_{n}-4 W W_{x} \mathcal{L}_{n} \\
= & \left(\partial_{x}-2 W\right)\left(\partial_{x x} \mathcal{L}_{n}+2 W \partial_{x} \mathcal{L}_{n}+2 W_{x} \mathcal{L}_{n}\right)
\end{aligned}
$$

So we get

$$
\left(\partial_{x}-2 W\right)\left\{\partial_{t_{2 n+1}} W+\left(\partial_{x x}+2 \partial_{x} W\right) \mathcal{L}_{n}\left[W_{x}-W^{2}\right]\right\}=0
$$

i.e., the MKdV hierarchy is

$$
\partial_{t_{2 n+1}} W+\partial_{x}\left(\partial_{x}+2 W\right) \mathcal{L}_{n}\left\{W_{x}-W^{2}\right\}=0 .
$$




\section{§2.3. The $\mathrm{P}_{\mathrm{II}}$ hierarchy}

In this section, we use the notation $\mathcal{L}_{n}$ to mean the operator defined by (1.4a) in the variables $U, x$, while $\hat{\mathcal{L}}_{n}$ denotes the usage of $\mathcal{L}_{n}$ with respective variables $V_{z}-V^{2}$ and $z$. Substituting

$$
W\left(x, t_{3}\right)=\frac{V(z)}{\left(3 t_{3}\right)^{\frac{1}{3}}}, z=\frac{x}{\left(3 t_{3}\right)^{\frac{1}{3}}}
$$

in the MKdV equation

$$
W_{t_{3}}-6 W^{2} W_{x}+W_{x x x}=0
$$

we obtain

$$
-\frac{V}{(3 t)^{\frac{4}{3}}}-\frac{x}{\left(3 t_{3}\right)^{\frac{5}{3}}} V^{\prime}-6 \frac{V^{2}}{\left(3 t_{3}\right)^{\frac{2}{3}}} \cdot \frac{V^{\prime}}{(3 t)^{\frac{2}{3}}}+\frac{V^{\prime \prime \prime}}{(3 t)^{\frac{4}{3}}}=0
$$

which implies

$$
V^{\prime \prime \prime}=6 V^{2} V^{\prime}+z V^{\prime}+V .
$$

Integration with respect to $z$ leads to

$$
V^{\prime \prime}=2 V^{3}+z V+\alpha_{1}, \quad \alpha_{1} \text { constant }
$$

Now for the hierarchy, we use the substitutions:

$$
\begin{aligned}
& W\left(x, t_{2 n+1}\right)=\frac{V(z)}{\left((2 n+1) t_{2 n+1}\right)^{\frac{1}{2 n+1}}}, \quad z=\frac{x}{\left((2 n+1) t_{2 n+1}\right)^{\frac{1}{2 n+1}}}, \\
& W_{x}-W^{2}=\frac{1}{\left((2 n+1) t_{2 n+1}\right)^{\frac{2}{2 n+1}}}\left(V^{\prime}-V^{2}\right) \\
& W_{t_{2 n+1}}=-\frac{V}{\left((2 n+1) t_{2 n+1}\right)^{\frac{2 n+2}{2 n+1}}}-\frac{z V^{\prime}}{\left((2 n+1) t_{2 n+1}\right)^{\frac{2 n+2}{2 n+1}}} .
\end{aligned}
$$


Note that by the above substitutions (2.3) and (2.4) we obtain

$$
\begin{gathered}
\mathcal{L}_{1}[U]=U=W_{x}-W^{2}=\frac{1}{((2 n+1) t)^{\frac{2}{2 n+1}}}\left(V^{\prime}-V^{2}\right) \\
=\frac{1}{((2 n+1) t)^{\frac{2}{2 n+1}}} \mathcal{L}_{1}\left[V^{\prime}-V^{2}\right] \\
\mathcal{L}_{2}[U]=U_{x x}+3 U^{2}=\frac{1}{((2 n+1) t)^{\frac{4}{2 n+1}}}\left\{\partial_{z z}\left(V^{\prime}-V^{2}\right)+3\left(V^{\prime}-V^{2}\right)^{2}\right\}
\end{gathered}
$$

\section{Lemma 2.1.}

$$
\mathcal{L}_{k}[U]=\frac{1}{((2 n+1) t)^{\frac{2 k}{2 n+1}}} \hat{\mathcal{L}}_{k}\left[V^{\prime}-V^{2}\right]
$$

Proof. The proof is by induction. The case $k=1$ holds by above. The relations

$$
\partial_{x} \mathcal{L}_{k+1}[U]=\frac{1}{((2 n+1) t)^{\frac{1}{2 n+1}}} \partial_{z} \mathcal{L}_{k+1}[U]
$$

and

$$
\partial_{x}^{3}+4 U \partial_{x}+2 U_{x}=\frac{1}{((2 n+1) t)^{\frac{3}{2 n+1}}}\left\{\partial_{z}^{3}+4\left(V^{\prime}-V^{2}\right) \partial_{z}+2\left(V^{\prime}-V^{2}\right) z\right\}
$$

imply

$$
\begin{aligned}
\partial_{z} \mathcal{L}_{k+1} & =\frac{1}{((2 n+1) t)^{\frac{2}{2 n+1}}}\left(\partial_{z}^{3}+4\left(V^{\prime}-V^{2}\right) \partial_{z}+2\left(V^{\prime}-V^{2}\right)_{z}\right) \frac{\hat{\mathcal{L}}_{k}}{((2 n+1) t)^{\frac{2 k}{2 n+1}}} \\
& =\frac{1}{((2 n+1) t)^{\frac{2(k+1)}{2 n+1}}}\left(\partial_{z}^{3}+4\left(V^{\prime}-V^{2}\right) \partial_{z}+2\left(V^{\prime}-V^{2}\right)_{z}\right) \hat{\mathcal{L}}_{k} .
\end{aligned}
$$


Therefore, we get the reduced hierarchy

$$
\begin{gathered}
-\frac{V}{\left((2 n+1) t_{2 n+1}\right)^{\frac{2(n+1)}{2 n+1}}}-\frac{z V^{\prime}}{\left((2 n+1) t_{2 n+1}\right)^{\frac{2(n+1)}{2 n+1}}}+\frac{1}{\left((2 n+1) t_{2 n+1}\right)^{\frac{2}{2 n+1}}} \\
\times \frac{1}{\left((2 n+1) t_{2 n+1}\right)^{\frac{2 n}{2 n+1}}} \frac{d}{d z}\left(\frac{d}{d z}+2 W\right) \hat{\mathcal{L}}_{n}\left[V^{\prime}-V^{2}\right]=0
\end{gathered}
$$

This gives the second Painlevé hierarchy

$$
\left(\frac{d}{d z}+2 V\right) \hat{\mathcal{L}}_{n}\left[V^{\prime}-V^{2}\right]=z V+\alpha_{n}, \quad n \geq 1 .
$$

Note that the case $n=0$ leads to an algebraic result

$$
(z-1) V=-\alpha_{0} \Rightarrow V=-\frac{\alpha_{0}}{z-1}
$$

which is not a DE. The next case $n=1$ is

$$
\left(\frac{d}{d z}+2 V\right)\left(V^{\prime}-V^{2}\right)=z V+\alpha_{1}
$$

which yields $\mathrm{P}_{\mathrm{II}}$ :

$$
V^{\prime \prime}=2 V^{3}+z V+\alpha_{1}
$$

The case $n=2$ is

$$
\left(\frac{d}{d z}+2 V\right)\left(\left(V^{\prime}-V^{2}\right)^{\prime \prime}+3\left(V^{\prime}-V^{2}\right)^{2}\right)=z V+\alpha_{2}
$$

which is equivalent to

$$
\begin{aligned}
V^{\prime \prime \prime \prime}-\left(2 V V^{\prime}\right)^{\prime \prime} & +6\left(V^{\prime}-V^{2}\right)\left(V^{\prime \prime}-2 V V^{\prime}\right) \\
+ & 2 V\left(V^{\prime \prime \prime}-2 V^{\prime 2}-2 V V^{\prime \prime}\right)+6 V\left(V^{\prime}-V^{2}\right)^{2}=z V+\alpha_{2}
\end{aligned}
$$

or

$$
\begin{gathered}
V^{(4)}-2\left(3 V^{\prime \prime} V^{\prime}+V V^{\prime \prime \prime}\right)+6\left(V^{\prime} V^{\prime \prime}-2 V V^{\prime 2}-V^{2} V^{\prime \prime}+2 V^{3} V^{\prime}\right) \\
+2 V V^{\prime \prime \prime}-4 V V^{2}-4 V^{2} V^{\prime \prime}+6 V\left(V^{\prime 2}-2 V^{2} V^{\prime}+V^{4}\right) \\
=z V+\alpha_{2}
\end{gathered}
$$

The end result is

$$
V^{(4)}-10 V V^{\prime 2}-10 V^{2} V^{\prime \prime}+6 V^{5}=z V+\alpha_{2} .
$$




\section{§2.4. Linear problems}

The KdV hierarchy arises as the compatibility of

$$
\left\{\begin{array}{l}
\eta_{x x}+(\lambda+U(x, \tau)) \eta=0 \\
\eta_{\tau}=A[\lambda ; U] \eta-B[\lambda ; U] \eta_{x},
\end{array}\right.
$$

for each time variable $\tau$. Note that this implies

$$
\begin{aligned}
- & \left\{\partial_{\tau} U \eta+(\lambda+U)\left(A \eta-B \eta_{x}\right)\right\} \\
= & A_{x x} \eta+2 A_{x} \eta_{x}+A(-\lambda-U) \eta \\
& \quad-B_{x x} \eta_{x}+2 B_{x}(\lambda+U) \eta+B\left(\lambda \eta_{x}+U_{x} \eta+U \eta_{x}\right)
\end{aligned}
$$

or, collecting terms

$$
\begin{aligned}
& \left(A_{x x}-(\lambda+U) A+2 B_{x}(\lambda+U)+B U_{x}+U_{\tau}+A(\lambda+U)\right) \eta \\
& \quad+\left(2 A_{x}-B_{x x}+\lambda B+U B-B(\lambda+U)\right) \eta_{x}=0 .
\end{aligned}
$$

Setting coefficients of $\eta$ and $\eta_{x}$ to zero, we find

$$
A=\frac{1}{2} B_{x}+a_{0}
$$

and

$$
U_{\tau}+\frac{1}{2}\left(\partial_{x}^{3}+4 U \partial_{x}+2 U_{x}\right) B+2 \lambda \partial_{x} B=0 .
$$

We take $B=B_{0} \lambda^{n}+B_{1} \lambda^{n-1}+\cdots+B_{n}$. These equations must hold identically in $\lambda$. Therefore, equating coefficients at each power of $\lambda$ leads to the following results.

$$
\lambda^{n+1}: \partial_{x} B_{0}=0,
$$

without loss of generality (by scaling $\lambda$ and $U, x, \tau$ if necessary), we assume $B_{0}=-1$ and for $0 \leq k \leq n-1$

$$
\lambda^{n-k}: \frac{1}{2}\left(\partial_{x}^{3}+4 U \partial_{x}+2 U_{x}\right) B_{k}=-2 \partial_{x} B_{k+1}
$$

and

$$
\lambda^{0}: U_{\tau}+\frac{1}{2}\left(\partial_{x}^{3}+4 U \partial_{x}+2 U_{x}\right) B_{n}=0
$$

That is,

$$
U_{\tau}-2 \partial_{x} B_{n+1}=0 .
$$


Comparing to the recursion operator $\mathcal{L}$, we have

$$
B_{k}[U]=-2\left(\frac{-1}{4}\right)^{k} \mathcal{L}_{k}[U], \quad k \geq 0\left(\text { Note: } B_{0}=-2 \mathcal{L}_{0}\right) .
$$

This means

$$
U_{\tau}-2 \cdot-2\left(-\frac{1}{4}\right)^{n+1} \partial_{x} \mathcal{L}_{n+1}[U]=0
$$

which implies

$$
U_{\tau}+\frac{-1}{(-4)^{n}} \partial_{x} \mathcal{L}_{n+1}[U]=0 .
$$

We recover the KdV hierarchy in the usual form (2.1) if we define

$$
\tau=-(-4)^{n} t_{2 n+1} .
$$

Or in the original notation for the hierarchy, we get

$$
\begin{gathered}
\left\{\begin{array}{c}
\eta_{x x}+\left(\lambda+U\left(x, t_{2 n+1}\right)\right) \eta=0 \\
\frac{-\eta_{t_{2 n+1}}}{(-4)^{n}}=\left[-\partial_{x}\left(\sum_{k=0}^{n} \frac{\mathcal{L}_{k}[U]}{(-4)^{k}} \lambda^{n-k}\right)+a_{0}\right] \eta \\
+2\left(\sum_{k=0}^{n} \frac{\mathcal{L}_{k}[U]}{(-4)^{k}} \lambda^{n-k}\right) \eta_{x}
\end{array}\right. \\
\text { i.e. }\left\{\begin{array}{c}
\eta_{x x}+\left(\lambda+U\left(x, t_{2 n+1}\right)\right) \eta=0 \\
\eta_{t_{2 n+1}}=\left[\partial_{x}\left(\sum_{k=0}^{n} \mathcal{L}_{k}[U](-4 \lambda)^{n-k}\right)+a_{n}\right] \eta \\
-2 \sum_{k=0}^{n} \mathcal{L}_{k}[U](-4 \lambda)^{n-k} \cdot \eta_{x} \\
a_{n}=-(-4)^{n} a_{0}
\end{array}\right.
\end{gathered}
$$

We show in Appendix A that this is a compatible system giving rise to the $\mathrm{KdV}$ hierarchy as its compatibility conditions.

\section{§2.5. Reduced linear problem}

The symmetry reduction (2.3) of the MKdV hierarchy implies a reduction of the KdV's linear problem through

$$
\eta\left(x, t_{2 n+1}\right)=\psi(z, s), \quad z=\frac{x}{\left((2 n+1) t_{2 n+1}\right)^{\frac{1}{2 n+1}}}, \quad s=\lambda\left((2 n+1) t_{2 n+1}\right)^{\frac{2}{2 n+1}} .
$$


In turn, these imply

$$
\begin{aligned}
\eta_{x x} & =\frac{1}{\left((2 n+1) t_{2 n+1}\right)^{\frac{2}{2 n+1}}} \psi_{z z} \\
\partial_{t_{2 n+1}} & =\frac{-x}{\left((2 n+1) t_{2 n+1}\right)^{\frac{2(n+1)}{2 n+1}}} \partial_{z}+2 \lambda\left((2 n+1) t_{2 n+1}\right)^{-\frac{2 n+1}{2 n+1}} \partial_{s} \\
& =\frac{1}{\left((2 n+1) t_{2 n+1}\right)^{\frac{2 n+1}{2 n+1}}}\left\{-z \partial_{z}+2 s \partial_{s}\right\} .
\end{aligned}
$$

Using the reduction (2.4) of the Miura transformation and recalling

$$
\mathcal{L}_{k}[U]=\frac{1}{\left((2 n+1) t_{2 n+1}\right)^{\frac{2 k}{2 n+1}}} \hat{\mathcal{L}}_{k}\left[V^{\prime}-V^{2}\right]
$$

that is,

$$
\mathcal{L}_{k}[U](-4 \lambda)^{n-k}=\frac{1}{\left((2 n+1) t_{2 n+1}\right)^{\frac{2 k}{2 n+1} \cdot \frac{2(n-k)}{2 n+1}}}(-4 s)^{n-k} \hat{\mathcal{L}}_{k}\left[V^{\prime}-V^{2}\right],
$$

we get

$$
\left\{\begin{array}{r}
\psi_{z z}+\left(s+V^{\prime}-V^{2}\right) \psi=0 \\
2 s \psi_{s}-z \psi_{z}=\left[\frac{d}{d z}\left(\sum_{k=0}^{n} \hat{\mathcal{L}}_{k}\left[V^{\prime}-V^{2}\right](-4 s)^{n-k}\right)+b_{n}\right] \psi \\
-2 \sum_{k=0}^{n} \hat{\mathcal{L}}_{k}\left[V^{\prime}-V^{2}\right](-4 s)^{n-k} \cdot \psi_{z} \\
b_{n}=a_{n}(2 n+1) t_{2 n+1}
\end{array}\right.
$$

(It is conventional at this point to take $s=\zeta^{2}$ and hence $2 s \partial_{s}=\frac{2 s}{2 \sqrt{s}} \partial_{\zeta}=\zeta \partial_{\zeta}$.)

We rewrite this as a system of $2 \times 2$ linear equations. Let $\varphi_{1}=\psi, \varphi_{2}=\psi_{z}$.

$$
\begin{aligned}
& \varphi_{z}=\left(\begin{array}{c}
\psi_{z} \\
\psi_{z z}
\end{array}\right)=\left(\begin{array}{cc}
0 & 1 \\
-\left(s+V^{\prime}-V^{2}\right) & 0
\end{array}\right)\left(\begin{array}{c}
\psi \\
\psi_{z}
\end{array}\right)=\left(\begin{array}{cc}
0 & 1 \\
-\left(s+V^{\prime}-V^{2}\right) & 0
\end{array}\right) \varphi \\
& \varphi_{s}=\left(\begin{array}{c}
\psi_{s} \\
\psi_{s z}
\end{array}\right)=\left(\begin{array}{ll}
A & B \\
C & D
\end{array}\right)\left(\begin{array}{c}
\psi \\
\psi_{z}
\end{array}\right)=\left(\begin{array}{ll}
A & B \\
C & D
\end{array}\right) \varphi
\end{aligned}
$$


where

$$
\begin{aligned}
A= & \frac{1}{2 s} \frac{d}{d z}\left(\sum_{k=0}^{n} \hat{\mathcal{L}}_{k}\left[V^{\prime}-V^{2}\right](-4 s)^{n-k}\right)+\frac{b_{n}}{2 s} \\
B= & \frac{z}{2 s}-\frac{1}{s} \sum_{k=0}^{n} \hat{\mathcal{L}}_{k}\left[V^{\prime}-V^{2}\right](-4 s)^{n-k} \\
C= & \left(\frac{z}{2 s}-\frac{1}{s} \sum_{k=0}^{n} \hat{\mathcal{L}}_{k}\left[V^{\prime}-V^{2}\right](-4 s)^{n-k}\right)\left(-s-V^{\prime}+V^{2}\right) \\
& +\frac{1}{2 s} \frac{d^{2}}{d z^{2}}\left(\sum_{k=0}^{n} \hat{\mathcal{L}}_{k}\left[V^{\prime}-V^{2}\right](-4 s)^{n-k}\right) \\
D= & \frac{1}{2 s}-\frac{1}{2 s} \frac{d}{d z}\left(\sum_{k=0}^{n} \hat{\mathcal{L}}_{k}\left[V^{\prime}-V^{2}\right](-4 s)^{n-k}\right)+\frac{b_{n}}{2 s} .
\end{aligned}
$$

For compatibility, see Appendix B.

\section{$\S 3 . \quad$ The Large-Parameter Limit of $\mathrm{P}_{\mathrm{II}}^{(n)}$}

In this section, we show that, for bounded $|z|$, the limit $\alpha_{n} \rightarrow \infty$ of $\mathrm{P}_{\mathrm{II}}^{(n)}$ is solved precisely by the stationary solutions of the $\mathrm{KdV}$ hierarchy. In this limit, $\mathrm{P}_{\mathrm{II}}^{(n)}$ becomes an autonomous hierarchy. The large-parameter limit of $\mathrm{P}_{\mathrm{II}}$ (for bounded $z$ ) was studied in [12]. The results are analogous to the $|z| \rightarrow \infty$ limit, first studied in 1913 by Boutroux [3]. The simultaneous limit $\alpha \rightarrow \infty$ and $|z| \rightarrow \infty$ of $\mathrm{P}_{\text {II }}$ has been studied extensively by Kawai, Takei et al. [14]-[16] and by Kitaev [17]. Asymptotic results as $|z| \rightarrow \infty$ for special classes of solutions of the whole $\mathrm{P}_{\mathrm{II}}^{(n)}$ hierarchy, called the tritronquée solutions, were obtained in [13].

Proposition 3.1. For each $n \geq 1$, the transformation

$$
V(z)=\alpha_{n}{ }^{1 /(2 n+1)} v(\zeta), \quad z=\alpha_{n}{ }^{-1 /(2 n+1)} \zeta
$$

maps $\mathrm{P}_{\mathrm{II}}^{(n)}$ to

$$
\left(\frac{d}{d \zeta}+2 v\right) \mathcal{L}_{n}\left\{v_{\zeta}-v^{2}\right\}=1+\frac{\zeta v}{\alpha_{n}},
$$

where $\mathcal{L}_{n}$ is now written in the variables $\zeta, v(\zeta)$.

Proof. The proof is by induction. The case $n=1$ follows from

$$
\mathcal{L}_{1}\left\{V_{z}-V^{2}\right\}=V_{z}-V^{2}=\alpha_{1}^{2 / 3}\left(v_{\zeta}-v^{2}\right)=\alpha_{1}^{2 / 3} \mathcal{L}_{1}\left\{v_{\zeta}-v^{2}\right\}
$$


and

$$
\left(\frac{d}{d z}+2 V\right)=\alpha_{1}^{1 / 3}\left(\frac{d}{d \zeta}+2 v\right)
$$

which implies that $\mathrm{P}_{\mathrm{II}}$ becomes

$$
\alpha_{1}\left(\frac{d}{d \zeta}+2 v\right) \mathcal{L}_{1}\left\{v_{\zeta}-v^{2}\right\}=\zeta v+\alpha_{1},
$$

as desired. For the inductive step, we use the notation $U=V_{z}-V^{2}, u=$ $v_{\zeta}-v^{2}$, and note that $U(z)=\alpha_{n}{ }^{2 /(2 n+1)} u(\zeta)$. We claim that the operator $\mathcal{L}_{n}$ transforms according to

$$
\mathcal{L}_{n}\{U\}=\alpha_{n}{ }^{p(n) /(2 n+1)} \mathcal{L}_{n}\{u\}, \quad p(n)=2 n .
$$

This result follows from the recursion relation (1.4a) along with (1.4b) which implies

$$
p(n+1)+1=p(n)+3, \quad p(1)=2 .
$$

Using the transformation (3.3), we get the desired result.

Remark 3.2. For each integer $n \geq 1$, and bounded $\zeta$, the solutions of $\mathrm{P}_{\mathrm{II}}^{(n)}$ in the limit $\alpha_{n} \rightarrow \infty$ satisfy

$$
\left(\frac{d}{d \zeta}+2 v\right) \mathcal{L}_{n}\left\{v_{\zeta}-v^{2}\right\}=1
$$

For $n=1,2$, these are

$$
\begin{aligned}
v^{\prime \prime} & =2 v^{3}+1 \\
v^{(4)} & =10 v v^{\prime 2}+10 v^{2} v^{\prime \prime}-6 v^{5}+1
\end{aligned}
$$

where the primes now refer to $\zeta$ derivatives.

Equation (3.4) is a first integral of the stationary version of the MKdV hierarchy (2.2). From Section 2, we know that any solution of the MKdV hierarchy must give a solution of the KdV hierarchy through the Miura transformation $U=W_{x}-W^{2}$. It is not widely known that for ODE reductions of these hierarchies, the Miura transformation is invertible. We recall here the invertible transformations between $\mathrm{P}_{\mathrm{II}}^{(n)}$ and the so-called thirty-fourth Painlevé hierarchy $\mathrm{P}_{34}^{(n)}$ given in [4]. 
Proposition 3.3. $\quad$ Suppose integer $n \geq 1$ is given. If $\mathcal{L}_{n}\{U\}-z / 2 \neq 0$, then

$$
\begin{aligned}
U & =V_{z}-V^{2} \\
V & =-\frac{1}{2 \mathcal{L}_{n}\{U\}-z}\left(\frac{d}{d z}\left(\mathcal{L}_{n}\{U\}\right)-\alpha_{n}\right)
\end{aligned}
$$

maps between the solutions $V(z)$ of $\mathrm{P}_{\mathrm{II}}^{(n)}$ and solutions $U(z)$ of

$$
\begin{aligned}
\mathrm{P}_{34}^{(n)}:\left(2 \mathcal{L}_{n}\{U\}\right. & -z) \frac{d^{2}}{d z^{2}}\left(\mathcal{L}_{n}\{U\}\right)-\left(\frac{d}{d z} \mathcal{L}_{n}\{U\}\right)^{2} \\
& +\frac{d}{d z} \mathcal{L}_{n}\{U\}+\left(2 \mathcal{L}_{n}\{U\}-z\right)^{2} U-\alpha_{n}\left(1-\alpha_{n}\right)=0 .
\end{aligned}
$$

For the case $n=1$, this is the well known transformation between $\mathrm{P}_{\mathrm{II}}$ and $\mathrm{P}_{34}$ (the thirty-fourth equation in the Painlevé classification given in Ince [10]). Note that differentiating $\mathrm{P}_{34}^{(n)}$ with respect to $z$ gives

$$
\frac{d}{d z} \mathcal{L}_{n+1}\{U\}=2 U+z U_{z}
$$

where we have used the recursion relation (1.4a). This is just the similarity reduction of the $\mathrm{KdV}$ hierarchy obtained via

$$
U\left(x, t_{2 n+1}\right)=\frac{U(z)}{\left[(2 n+1) t_{2 n+1}\right]^{2 /(2 n+1)}}, \quad z=\frac{x}{\left[(2 n+1) t_{2 n+1}\right]^{1 /(2 n+1)}} .
$$

Under the transformation (3.1), the mappings (3.7) become

$$
\begin{aligned}
& u=v_{\zeta}-v^{2} \\
& v=-\frac{1}{2 \mathcal{L}_{n}\{u\}-\zeta / \alpha_{n}}\left(\frac{d}{d z}\left(\mathcal{L}_{n}\{u\}\right)-1\right)
\end{aligned}
$$

Moreover, the differentiated hierarchy (3.9) becomes

$$
\frac{d}{d \zeta} \mathcal{L}_{n+1}\{u\}=\frac{1}{\alpha_{n}}\left(2 u+\zeta u_{\zeta}\right)
$$

Taking the limit $\alpha_{n} \rightarrow \infty$, we get the stationary KdV hierarchy

$$
\frac{d}{d \zeta} \mathcal{L}_{n+1}\{u\}=0
$$

Any solution $u(\zeta)$ of the $n$-th member of this hierarchy yields a solution $v(\zeta)$ of the $n$-th member of the limiting $\mathrm{P}_{\mathrm{II}}^{(n)}$ equation (3.4) under the mapping

$$
v=-\frac{1}{2 \mathcal{L}_{n}\{u\}}\left(\frac{d}{d z}\left(\mathcal{L}_{n}\{u\}\right)-1\right) .
$$


We show how to construct solutions of the stationary KdV hierarchy in the next section.

\section{$\S 4$. Stationary Solutions of the KdV Hierarchy}

In this section, we review the construction of periodic solutions of the stationary KdV hierarchy. We follow a construction that was first given by Drach in 1919 [5]. Properties of these solutions were developed and studied in detail by the Russian school, see [6] and [20]. To simplify the notation, for each integer $n \geq 1$, we write $\tau$ instead of $t_{2 n+1}$ and use $U$ both for the solutions of the KdV hierarchy and its stationary version.

Consider two independent solutions $\eta_{1}, \eta_{2}$ of the Schrödinger equation

$$
\eta_{x x}+(\lambda+U(x, \tau)) \eta=0
$$

which forms the first half of the linear problem (2.6). Clearly, the Wronskian $\omega:=\eta_{1}{ }^{\prime} \eta_{2}-\eta_{1} \eta_{2}{ }^{\prime}$ is constant in $x$. The product $R(x ; \lambda):=\eta_{1} \eta_{2}$ satisfies

$$
\begin{aligned}
R^{\prime \prime} & =-2(\lambda+U(x, \tau)) R+2 \eta_{1}{ }^{\prime} \eta_{2}{ }^{\prime} \\
& =-2(\lambda+U(x, \tau)) R+\frac{1}{2 R}\left(\left(\eta_{1}{ }^{\prime} \eta_{2}+\eta_{1}{\eta_{2}}^{\prime}\right)^{2}-\left(\eta_{1}{ }^{\prime} \eta_{2}-\eta_{1}{\eta_{2}}^{\prime}\right)^{2}\right) \\
& =-2(\lambda+U(x, \tau)) R+\frac{1}{2 R}\left({R^{\prime}}^{2}-\omega^{2}\right)
\end{aligned}
$$

where primes denote differentiation in $x$. In other words, $R$ satisfies

$$
2 R R^{\prime \prime}+4(\lambda+U(x, \tau)) R^{2}-{R^{\prime}}^{2}+\omega^{2}=0 .
$$

The solutions of the $\mathrm{KdV}$ that we seek correspond to products $R$ that are polynomial in $\lambda$. Equation (4.1) implies that, then, $\omega^{2}$ is also polynomial in $\lambda$.

We write

$$
R(x ; \lambda)=\prod_{k=1}^{n}\left(\gamma_{k}(x)-\lambda\right), \quad \Omega(\lambda):=\omega^{2}=-4 \prod_{i=1}^{2 n+1}\left(\lambda-\lambda_{i}\right),
$$

where we have used the highest degree terms in Equation (4.1) to relate the degree of $\omega^{2}$ to the degree of $R$ and their respective coefficients. If we evaluate Equation (4.1) at each zero $\gamma_{j}(x)$ of $R$, we are led to the system of equations

$$
\gamma_{j}{ }^{\prime}(x)^{2} \prod_{k=1, k \neq j}^{n}\left(\gamma_{k}(x)-\gamma_{j}(x)\right)^{2}=-4 \prod_{i=1}^{2 n+1}\left(\gamma_{j}(x)-\lambda_{i}\right), 1 \leq j \leq n .
$$


Moreover, the coefficients of $\lambda^{2 n}$ give

$$
U(x, \tau)=2 \sum_{k=1}^{n} \gamma_{k}(x, \tau)-\sum_{i=1}^{2 n+1} \lambda_{i} .
$$

Consider now the second part of the linear problem (2.9). The result of differentiating $R$ with respect to $\tau$ is

$$
\begin{aligned}
R_{\tau} & =\eta_{1_{\tau}} \eta_{2}+\eta_{1} \eta_{2 \tau} \\
& =\left(A \eta_{1}-B \eta_{1_{x}}\right) \eta_{2}+\eta_{1}\left(A \eta_{2}-B \eta_{2 x}\right) \\
& =2 A R-B R_{x}
\end{aligned}
$$

Similarly, we get

$$
\begin{aligned}
\omega_{\tau} & =\left(2 A-B_{x}\right) \omega \\
& =2 a_{0} \omega
\end{aligned}
$$

where we have used Equation (2.7). For the stationary case, it is clear that we must take $a_{0}=0$. In that case, with $R_{\tau}=0$, Equation (4.4) gives

$$
B_{x} R-B R_{x}=0 \quad \Rightarrow \quad B=\beta R
$$

where $\beta$ is a constant.

To be more explicit, we consider the special cases $n=1$ and $n=2$. In the case $n=1$, Equation (4.1) leads to

$$
\begin{aligned}
& \lambda^{2}: \gamma_{1}(x)=\frac{1}{2}\left(\lambda_{1}+\lambda_{2}+\lambda_{3}+U(x)\right) \\
& \lambda: \gamma_{1}{ }^{\prime \prime}(x)=2 \gamma_{1}(x)^{2}-2\left(\lambda_{1} \lambda_{2}+\lambda_{1} \lambda_{3}+\lambda_{2} \lambda_{3}+2 U(x) \gamma_{1}(x)\right) \\
& 1: \gamma_{1}(x) \gamma_{1}{ }^{\prime \prime}(x)=\frac{1}{2} \gamma_{1}{ }^{\prime}(x)^{2}-2 U(x) \gamma_{1}(x)^{2}-2 \lambda_{1} \lambda_{2} \lambda_{3}
\end{aligned}
$$

Multiplying Equation (4.7) by $\gamma_{1}(x)$ and subtracting it from Equation (4.8), then substituting for $U$ by using Equation (4.6), gives

$$
\gamma_{1}^{\prime}(x)^{2}=-4\left(\lambda_{1}-\gamma_{1}(x)\right)\left(\lambda_{2}-\gamma_{1}(x)\right)\left(\lambda_{3}-\gamma_{1}(x)\right)=\Omega\left(\gamma_{1}(x)\right) .
$$

This equation is solved by Weierstrass $\wp$ functions. The solution is given by the inversion of the function defined by

$$
\int^{\gamma_{1}} \frac{d s}{\sqrt{\Omega(s)}}=x+c_{0},
$$


where $c_{0}$ is a constant. Note that although the stationary KdV equation governing $U$ is third-order, there appears to be four arbitrary constants $\left(\lambda_{1}, \lambda_{2}, \lambda_{3}, c_{0}\right)$ in the description of the solution. To see that there must be one relation between them, we use Equation (4.5) and the results for $B$ from Section 2. In the case $n=1$, we get $B=-\lambda+U / 2=\beta\left(\gamma_{1}(x)-\lambda\right)$ which implies that $\beta=1$ and $U=2 \gamma_{1}$. That is,

$$
\lambda_{1}+\lambda_{2}+\lambda_{3}=0
$$

For the case $n=2$, we get

$$
\begin{aligned}
\gamma_{1}{ }^{\prime}(x)^{2} & =\frac{\Omega\left(\gamma_{1}(x)\right)}{\left(\gamma_{1}(x)-\gamma_{2}(x)\right)^{2}} \\
\gamma_{2}{ }^{\prime}(x)^{2} & =\frac{\Omega\left(\gamma_{2}(x)\right)}{\left(\gamma_{1}(x)-\gamma_{2}(x)\right)^{2}}
\end{aligned}
$$

where $\Omega\left(\gamma_{k}(x)\right)=-4\left(\lambda_{1}-\gamma_{k}(x)\right)\left(\lambda_{2}-\gamma_{k}(x)\right)\left(\lambda_{3}-\gamma_{k}(x)\right)\left(\lambda_{4}-\gamma_{k}(x)\right)\left(\lambda_{5}-\right.$ $\left.\gamma_{k}(x)\right)$. Equations (4.10) define hyperelliptic functions. By the Jacobi inversion theorem, the solutions are given by

$$
\begin{aligned}
& \int^{\gamma_{1}} \frac{d s}{\sqrt{\Omega(s)}}+\int^{\gamma_{2}} \frac{d s}{\sqrt{\Omega(s)}}=c_{0} \\
& \int^{\gamma_{1}} \frac{s d s}{\sqrt{\Omega(s)}}+\int^{\gamma_{2}} \frac{s d s}{\sqrt{\Omega(s)}}=x+c_{1}
\end{aligned}
$$

where $c_{0}$ and $c_{1}$ are arbitrary constants. To prove that these are solutions of Equations (4.10), we note that differentiation of Equations (4.11) gives

$$
\begin{gathered}
\frac{\gamma_{1}{ }^{\prime}(x)}{\sqrt{\Omega\left(\gamma_{1}(x)\right)}}+\frac{\gamma_{2}{ }^{\prime}(x)}{\sqrt{\Omega\left(\gamma_{2}(x)\right)}}=0 \\
\frac{\gamma_{1}(x) \gamma_{1}{ }^{\prime}(x)}{\sqrt{\Omega\left(\gamma_{1}(x)\right)}}+\frac{\gamma_{2}(x) \gamma_{2}{ }^{\prime}(x)}{\sqrt{\Omega\left(\gamma_{2}(x)\right)}}=1
\end{gathered}
$$

Multiplying the first equation by $\gamma_{k}(x), k=1,2$ and subtracting the second equation from it, we get

$$
\frac{\left(\gamma_{1}(x)-\gamma_{2}(x)\right) \gamma_{k}{ }^{\prime}(x)}{\sqrt{\Omega\left(\gamma_{k}(x)\right)}}=1, \quad k=1,2
$$

which are equivalent to Equations (4.10). It is well known that the functions $\gamma_{k}(x)$ obtained by inversion of Equations (4.11) are multivalued functions, but their symmetric combinations $\gamma_{1}(x)+\gamma_{2}(x)$ and $\gamma_{1}(x) \gamma_{2}(x)$ are meromorphic. Hence the solution $U(x)$ of the stationary fifth-order KdV equation given by

$$
U(x)=2\left(\gamma_{1}(x)+\gamma_{2}(x)\right)-\left(\lambda_{1}+\lambda_{2}+\lambda_{3}+\lambda_{4}+\lambda_{5}\right)
$$


is meromorphic. Use of Equation (4.5) shows that now there are two conditions on the constants $\lambda_{i}$, namely

$$
\sum_{i=1}^{5} \lambda_{i}=0, \quad \sum_{\substack{i, j=1 \\ i \neq j}}^{5} \lambda_{i} \lambda_{j}=0 .
$$

To deduce solutions $u(\zeta)$ of the hierarchy Equation (3.11) in the limit $\alpha_{n} \rightarrow \infty$, we use Equation (4.3) with $U(x, \tau)$ replaced by $u(\zeta)$ and $\gamma_{k}(x, \tau)$ replaced by $\gamma_{k}(\zeta)$. Here the hyperelliptic functions $\gamma_{k}(\zeta)$ are given by inversions of

$$
\begin{aligned}
& \sum_{k=1}^{n} \int^{\gamma_{k}} \frac{s^{i} d s}{\sqrt{\Omega(s)}}=c_{i}, \quad i=0, \ldots, n-2, \\
& \sum_{k=1}^{n} \int^{\gamma_{k}} \frac{s^{n-1} d s}{\sqrt{\Omega(s)}}=\zeta+c_{n-1}
\end{aligned}
$$

where $c_{0}, \ldots, c_{n-1}$ are arbitrary constants and the numbers $\lambda_{i}, i=1, \ldots, 2 n+1$ satisfy $n$ conditions on their symmetric combinations.

\section{$\S 5 . \quad$ Summary}

In this paper, we gave an explicit review of the reduction of the $\mathrm{KdV}$ hierarchy to the $\mathrm{P}_{\mathrm{II}}$ hierarchy. We deduced this via the MKdV hierarchy and its symmetry reduction. We also explicitly described the Lax pair for the KdV hierarchy and its reduction to the linear problem for the $\mathrm{P}_{\mathrm{II}}$ hierarchy.

Many hierarchies may be associated with the same Painlevé equation. The reason is that reductions of many different soliton PDEs may give rise to the same Painlevé equation. Correspondingly, each PDE's hierarchy may reduce to a different hierarchy for that Painlevé equation. For example, $\mathrm{P}_{\mathrm{II}}$ is known to have at least two hierarchies, one given by the above reduction of the $\mathrm{KdV}$ equation [2] and another constructed from a water wave equation in [9] (see also [8]). However, we did not describe such alternative hierarchies in this paper.

Our main focus lay on methods of deducing explicit information about solutions of hierarchies. In particular, we reviewed the construction of periodic solutions of the stationary $\mathrm{KdV}$ equation, in order to find solutions of autonomous limits of the second Painlevé hierarchy.

Another motivation for this work was to fill the gap in our knowledge concerning the transcendental nature of the solutions of the higher-order members of a given Painlevé hierarchy. Painlevé's original mathematical investigations 
were motivated by a search for higher-transcendental functions, of greater transcendentality than the elliptic functions. The proof of transcendentality of the solutions of the second-order Painlevé equations relied on sophisticated applications of differential Galois theory [22]. To our knowledge, no attempt towards a proof has been made for higher-order cases. An outstanding question in the field is whether the solutions of higher-order Painlevé equations (in a hierarchy) not only give rise to yet higher transcendental functions, but whether these are of higher transcendental nature than the solutions of the second-order cases.

Asymptotic behaviours of the solutions of the higher-order Painlevé equations provide a start towards answering such questions. In this paper, we described the method of obtaining the hyperelliptic solutions of the stationary KdV hierarchy through its linear problem. We also showed that these solutions give rise to solutions of an autonomous hierarchy obtained as large parameter limits of the second Painlevé hierarchy. Since hyperelliptic functions can be considered to be higher transcendental functions than elliptic functions, such behaviours appear to imply that the solutions of higher-order members of the hierarchy must be of higher transcendental nature than those of the secondorder Painlevé equations.

\section{Acknowledgements}

The author would like to acknowledge informative discussions with Chris Cosgrove, Marta Mazzocco, Vladimir Novikov, and Andrew Pickering.

\section{Appendix A. Compatibility of KdV Hierarchy's Linear Problem}

Here we show that the linear problem (2.9) is compatible and that the compatibility conditions are precisely the equations of the $\mathrm{KdV}$ hierarchy.

$$
\begin{aligned}
-\frac{\partial U}{\partial t_{2 n+1}} \eta- & (\lambda+U)\left(\partial_{x}\left(\sum \mathcal{L}_{k}(-4 \lambda)^{n-k}\right)+a_{n}\right) \eta \\
& +2(\lambda+U)\left(\sum \mathcal{L}_{k}(-4 \lambda)^{n-k}\right) \eta_{x} \\
= & \partial_{x x x}\left(\sum \mathcal{L}_{k}(-4 \lambda)^{n-k}\right) \eta+2 \partial_{x x}\left(\sum \mathcal{L}_{k}(-4 \lambda)^{n-k}\right) \eta_{x} \\
& +\left[\partial_{x}\left(\sum \mathcal{L}_{k}(-4 \lambda)^{n-k}\right)+a_{n}\right]-(\lambda+U) \eta \\
& -2\left\{\partial_{x x}\left(\sum \mathcal{L}_{k}(-4 \lambda)^{n-k}\right) \eta_{x}+2 \partial_{x}\left(\sum \mathcal{L}_{k}(-4 \lambda)^{n-k}\right)\right. \\
& \left.-(\lambda+U) \eta+\left(\sum \mathcal{L}_{k}(-4 \lambda)^{n-k}\right) \cdot\left[-U_{x} \eta-(\lambda+U) \eta_{x}\right]\right\}
\end{aligned}
$$


Equating the coefficients of $\eta_{x}$ and $\eta$ to zero separately, we get

$$
\begin{aligned}
2(\lambda+ & U) \sum \mathcal{L}_{k}(-4 \lambda)^{n-k} \\
= & 2 \partial_{x x}\left(\sum \mathcal{L}_{k}(-4 \lambda)^{n-k}\right)-2 \partial_{x x} \sum \mathcal{L}_{k}(-4 \lambda)^{n-k} \\
& +2(\lambda+U) \sum \mathcal{L}_{k}(-4 \lambda)^{n-k},
\end{aligned}
$$

and

$$
\begin{gathered}
-\frac{\partial U}{\partial t_{2 n+1}}-(\lambda+U)\left[\partial_{x}\left(\sum \mathcal{L}_{k}(-4 \lambda)^{n-k}\right)+a_{n}\right] \\
=\partial_{x x x}\left(\sum \mathcal{L}_{k}(-4 \lambda)^{n-k}\right)-(\lambda+U)\left[\partial_{x}\left(\sum \mathcal{L}_{k}(-4 \lambda)^{n-k}\right)+a_{n}\right] \\
\quad+4(\lambda+U) \partial_{x}\left(\sum \mathcal{L}_{k}(-4 \lambda)^{n-k}\right)+2 U_{x}\left(\sum \mathcal{L}_{k}(-4 \lambda)^{n-k}\right) .
\end{gathered}
$$

Hence

$$
\begin{aligned}
-\frac{\partial U}{\partial t_{2 n+1}} & =\left(\partial_{x}^{3}+4 U \partial_{x}+2 U_{x}\right) \sum \mathcal{L}_{k}(-4 \lambda)^{n-k}-\partial_{x} \sum_{\substack{l=-1 \\
l=k-1}}^{n-1} \mathcal{L}_{l+1}(-4 \lambda)^{n-l} \\
& =\partial_{x} \sum_{k=0}^{n} \mathcal{L}_{k+1}(-4 \lambda)^{n-k}-\partial_{x} \sum_{l=0}^{n-1} \mathcal{L}_{l+1}(-4 \lambda)^{n-k} \\
& =\partial_{x} \mathcal{L}_{n+1}
\end{aligned}
$$

\section{Appendix B. Compatibility of Reduced Linear System}

In this appendix, we show that the reduced linear system (2.11) is compatible and that the compatibility conditions are precisely $\mathrm{P}_{\mathrm{II}}^{(n)}$. We first observe

$$
\begin{aligned}
& -\psi-\left(s+V^{\prime}-V^{2}\right)\left\{\frac{z}{2 s} \psi_{z}+\frac{1}{2 s}\left[\partial \sum \hat{\mathcal{L}}_{k}(-4 s)^{n-k}+b_{n}\right] \psi\right. \\
& \left.\quad-\frac{1}{s}\left(\sum \hat{\mathcal{L}}_{k}(-4 s)^{n-k}\right) \psi_{z}\right\} \\
& =\frac{1}{s} \psi_{z z}+\frac{z}{2 s} \psi_{z z z}+\frac{1}{2 s}\left[\partial_{z z z} \sum \hat{\mathcal{L}}_{k}(-4 s)^{n-k}\right] \psi \\
& \quad+\frac{1}{s} \partial_{z z}\left(\sum \hat{\mathcal{L}}_{k}(-4 s)^{n-k}\right) \psi_{z}+\frac{1}{2 s}\left(\partial_{z} \sum \hat{\mathcal{L}}_{k}(-4 s)^{n-k}+b_{n}\right) \psi_{z z} \\
& -\frac{1}{s} \partial_{z z}\left(\sum \hat{\mathcal{L}}_{k}(-4 s)^{n-k}\right) \psi_{z}-\frac{2}{s} \partial_{z} \sum \hat{\mathcal{L}}_{k}(-4 s)^{n-k} \psi_{z z} \\
& \quad-\frac{1}{s} \sum \hat{\mathcal{L}}_{k}(-4 s)^{n-k} \cdot \psi_{z z z}
\end{aligned}
$$




$$
\begin{aligned}
= & -\frac{1}{s}\left(s+V^{\prime}+V^{2}\right) \psi-\frac{z}{2 s}\left[\left(V^{\prime}-V^{2}\right)^{\prime} \psi+\left(s+V^{\prime}-V^{2}\right) \psi_{z}\right] \\
& +\frac{1}{2 s} \sum \partial_{z z z} \hat{\mathcal{L}}_{k}(-4 s)^{n-k} \cdot \psi+\frac{1}{s} \sum \partial_{z z} \hat{\mathcal{L}}_{k}(-4 s)^{n-k} \cdot \psi_{z} \\
& -\frac{1}{2 s}\left(\sum \partial_{z} \hat{\mathcal{L}}_{k}(-4 s)^{n-k}+b_{n}\right)\left(s+V^{\prime}-V^{2}\right) \psi \\
& -\frac{1}{s} \sum \partial_{z z} \hat{\mathcal{L}}_{k}(-4 s)^{n-k} \cdot \psi_{z} \\
& +\frac{2}{s} \sum \partial_{z} \hat{\mathcal{L}}_{k}(-4 s)^{n-k} \cdot\left(s+V^{\prime}-V^{2}\right) \psi \\
& +\frac{1}{s} \sum \hat{\mathcal{L}}_{k}(-4 s)^{n-k} \cdot\left[\left(V^{\prime}-V^{2}\right)^{\prime} \psi+\left(s+V^{\prime}-V^{2}\right) \psi_{z}\right]
\end{aligned}
$$

Note that the coefficients of $\psi_{z}$ cancel and we are left only with coefficients of $\psi$ which give

$$
\begin{aligned}
- & 1-\frac{b_{n}}{2 s}\left(s+V^{\prime}-V^{2}\right)-\frac{\left(s+V^{\prime}-V^{2}\right)}{2 s} \sum \partial_{z} \hat{\mathcal{L}}_{k}(-4 s)^{n-k} \\
= & -\frac{1}{s}\left(s+V^{\prime}-V^{2}\right)-\frac{z}{2 s}\left[\left(V^{\prime}-V^{2}\right)^{\prime}\right] \\
& +\frac{1}{2 s} \sum\left(\partial_{z}^{3}+3\left(V^{\prime}-V^{2}\right) \partial_{z}+2\left(V^{\prime}-V^{2}\right)^{\prime}\right) \hat{\mathcal{L}}_{k}(-4 s)^{n-k} \\
& -\frac{1}{2} \sum \partial_{z} \hat{\mathcal{L}}_{k}(-4 s)^{n-k}-\frac{b_{n}}{2 s}\left(2+V^{\prime}-V^{2}\right)+2 \sum \partial_{z} \hat{\mathcal{L}}_{k}(-4 s)^{n-k}
\end{aligned}
$$

This implies

$$
\begin{aligned}
\sum & {\left[\partial_{z}^{s}+4\left(V^{\prime}-V^{2}\right) \partial_{z}+2\left(V^{\prime}-V^{2}\right)^{\prime}\right] \hat{\mathcal{L}}_{k}(-4 s)^{n-k} } \\
& -\sum_{l=-1}^{n-1} \partial_{z} \mathcal{L}_{l+1}(-4 s)^{n-l}-2\left(V^{\prime}-V^{2}\right)-z\left(V^{\prime}-V^{2}\right)^{\prime}=0
\end{aligned}
$$

Hence

$$
\begin{aligned}
& \sum_{k=0}^{n} \partial_{z} \hat{\mathcal{L}}_{k+1}(-4 s)^{n-k}-\sum_{l=0}^{n-1} \partial_{z} \hat{\mathcal{L}}_{l+1}(-4 s)^{n-l} \\
& \quad-2\left(V^{\prime}-V^{2}\right)-z\left(V^{\prime}-V^{2}\right)^{\prime}=0
\end{aligned}
$$

that is,

$$
\partial_{z} \hat{\mathcal{L}}_{n+1}-2\left(V^{\prime}-V^{2}\right)-z\left(V^{\prime}-V^{2}\right)=0 .
$$

Recalling

$$
\begin{aligned}
\partial_{z} \hat{\mathcal{L}}_{n} & =\left[\partial_{z}^{3}+4\left(V^{\prime}-V^{2}\right) \partial_{z}+2\left(V^{\prime \prime}-2 V V^{\prime}\right)\right] \hat{\mathcal{L}}_{n} \\
& =\left(\partial_{z}-2 V\right)\left(\partial_{z}^{2}+2 V \partial_{z}+2 V^{\prime}\right) \hat{\mathcal{L}}_{n},
\end{aligned}
$$


we thus obtain

$$
\left(\partial_{z}-2 V\right) \partial_{z}\left(\partial_{z}+2 V\right) \hat{\mathcal{L}}_{n}-\left(\partial_{z}-2 V\right)\left(V+2 V^{\prime}\right)=0
$$

that is,

$$
\partial_{z}\left(\partial_{z}+2 V\right) \hat{\mathcal{L}}_{n}-\partial_{z}\left(z V+\alpha_{n}\right)=0 .
$$

\section{References}

[1] Ablowitz, M. J. and Clarkson, P. A., Solitons, Nonlinear Evolution Equations, and Inverse Scattering, Cambridge University Press, Cambridge; New York, 1991.

[2] Airault, H., Rational solutions of Painlevé equations, Stud. Appl. Math., 61 (1979), 31-53.

[3] Boutroux, P., Recherches sur les transcendantes de M. Painlevé et l'étude asymptotique des équations différentielles du second ordre, Ann. École Norm., 30 (1913), 265-375; 31 (1914), 99-159.

[4] Clarkson, P. A., Joshi, N. and Pickering, A., Bäcklund transformations for the second Painlevé hierarchy: a modified truncation approach, Inverse Problems, 15 (1999), 175187.

[5] Drach, J., Appendix II: Sur l'integration par quadratures de l'équation $d^{2} y / d x^{2}=$ $[\varphi(x)+h] y$, Compte Rendus l'Acad Sci., 7 (1919), 337-340.

[6] Dubrovin, B. A., Theta functions and non-linear equations, Russian Math. Surveys, 36 (1981), 11-91, translated from Uspekhi Mat. Nauk., 36 (1981), 81-140.

[7] Fokas, A. S. and Zhou, X., On the solvability of Painlevé II and IV, Comm. Math. Phys., 144 (1992), 601-622.

[8] Gordoa, P. R. and Pickering, A., Nonisospectral scattering problems: A key to integrable hierarchies, J. Math. Phys., 40 (1999), 5749-5786.

[9] Gordoa, P. R., Joshi, N. and Pickering, A., On a generalized $2+1$ dispersive water wave hierarchy, Publ. RIMS, Kyoto Univ., 37 (2001), 327-347.

[10] Ince, E. L., Ordinary Differential Equations Longmans, Green and Co., London and New York, 1927 [Reprinted (Dover, New York, 1956)].

[11] Joshi, N. and Kruskal, M. D., The Painlevé connection problem: an asymptotic approach I, Stud. Appl. Math., 86 (1992), 315-376.

[12] Joshi, N., The second Painlevé equation in the large parameter limit I: local asymptotic analysis, Stud. Appl. Math., 102 (1999), 345-373.

[13] Joshi, N. and Mazzocco, M., Existence and uniqueness of tri-tronquée solutions of the second Painlevé hierarchy, Nonlinearity, 16 (2003), 427-439.

[14] Kawai, T. and Takei, Y., WKB analysis of Painlevé transcendents with a large parameter. I, Adv. Math., 118 (1996), 1-33.

[15] Aoki, T., Kawai, T. and Takei, Y., WKB analysis of Painlevé transcendents with a large parameter. II. Multiple-scale analysis of Painlevé transcendents, in Structure of solutions of differential equations, (Katata/Kyoto, 1995), World Sci. Publishing, River Edge, NJ, pp. 1-49, 1996.

[16] Kawai, T. and Takei, Y., WKB analysis of Painlevé transcendents with a large parameter. III. Local reduction of 2-parameter Painlevé transcendents, Adv. Math., 134 (1998), 178-218.

[17] Kitaev, A. V., Turning points of linear systems and double asymptotics of the Painlevé transcendents, in Painlevé transcendents (Sainte-Adèle, PQ, 1990), NATO Adv. Sci. Inst. Ser. B Phys. 278 Plenum, New York, (1992), 81-96. 
[18] Lax, P. D, Almost periodic solutions of the KdV equation, SIAM review, 18(3) (1976), 351-375.

[19] A Hamiltonian approach to the KdV equation, Publ. Math. Res. Center Univ. Wisconsin, Academic Press, New York-London, 40 (1978), 207-224.

[20] Novikov, S., Manakov, S. V., Pitaevskii, L. P. and Zakharov, V. E., Theory of Solitons, the inverse scattering method (translated from Teoriia Solitonov). Consultants Bureau; New York and London, 1984.

[21] Painlevé, P., Sur les Equations Differentielles du Second Ordre et d'Ordre Superieur, dont l'Interable Generale est Uniforme, Acta Math., 25 (1902), 1-86.

[22] Umemura, H. and Watanabe, H., Solutions of the second and fourth Painlevé equations I, Nagoya Math. J., 148 (1997), 151-198. 